\title{
LA INDUSTRIA Y LA CUALIFICACION PROFESIONAL DE LOS TRABAJADORES
}

\author{
Eugenio A. CLIMENT LOPEZ \\ Departamento de Geografía y Ordenación del Territorio \\ Universidad de Zaragoza
}

\begin{abstract}
Resumen: La producción industrial se organiza según el modelo de especialización flexible, que se manifiesta en el mercado laboral como flexibilidad numérica y funcional; esta última requiere una elevada cualificación profesional de los trabajadores, siendo un factor clave de localización industrial. En este artículo se estudian las actitudes y estrategias de las empresas industriales españolas respecto a la cualificación y la formación profesionales.
\end{abstract}

Palabras clave: Localización industrial, cualificación profesional, formación profesional.

Abstract: Industrial production is organized according to the flexible specialization pattern, that is manifested on labour market as numerical and functional flexibility; the last one demands a high professional qualification of the workers, being a key factor of industrial location. In this article are studied the spanish industrial firms attitudes and strategies on professional qualification and education.

Key words: Industrial location, professional qualification, professional education.

\section{INTRODUCCION}

La mejora de la cualificación profesional de los trabajadores ha pasado a ser una de las preocupaciones más destacadas de la sociedad española: el gobierno, por una parte, viene desarrollando a partir de la ley 1/1990, de ordenación general del sistema educativo (LOGSE), una reforma de la educación, en la que la formación 
profesional es el nivel de estudios que más se intenta revalorizar y en el que más novedades se introducen. Las organizaciones empresariales y los sindicatos, por otra parte, han mostrado una especial sensibilidad ante dicha mejora, puesta de manifiesto en el hecho de que haya pasado a ser uno de los elementos tomados en consideración en la negociación colectiva y en el conjunto de las relaciones laborales.

Esta preocupación de la sociedad española está en consonancia con la del conjunto de la Unión Europea, en el seno de cuya Comisión viene funcionando desde hace tiempo un grupo de trabajo especializado en recursos humanos, educación, formación y juventud, que impulsa y dirige una serie de programas, Erasmus y Petra entre ellos.

Este artículo tiene un doble objetivo: por una parte, estructurar algunas reflexiones teóricas acerca de la cualificación profesional como factor de localización de la industria, en el contexto de la dinámica económica actual. Por otra parte, poner de manifiesto y valorar, a la luz de las mismas, las actitudes de las empresas industriales respecto a la cualificación profesional de sus empleados y las estrategias que adoptan de cara a la formación de los mismos, tomando como punto de partida para ello un estudio propio sobre La Rioja, cuyas conclusiones, una vez contrastadas con las de otros estudios, pueden generalizarse al conjunto del valle del Ebro y de España.

\section{ESPECIALIZACION FLEXIBLE Y CUALIFICACION PROFESIONAL}

Desde que la economía mundial entró, a principios de los años setenta, én la fase descendente del ciclo económico iniciado tras la segunda guerra mundial, ciclo que puede interpretarse como la cuarta onda de Kondratieff (HALL y PRESTON, 1990), se viene constatando la crisis del fordismo como modelo de organización de la industria.

El fordismo, materializado a partir de finales del siglo $\mathrm{XIX}$, consiste en organizar la producción industrial en base a factorias y empresas de gran tamaño, resultantes de un proceso continuado de concentración de capital. La competitividad de estas empresas reside en las economías de escala que se obtienen con la fabricación en serie de productos estandarizados por medio de cadenas de producción; éstas son el resultado de llevar a sus últimas consecuencias el principio de la división del trabajo, junto con la utilización masiva de bienes de equipo basados en la entonces nueva tecnología electromecánica.

Desde principios de los años setenta, como en toda fase recesiva, la competencia entre las empresas industriales se hace más dura, en un contexto económico definido en lo esencial por dos procesos: primero, la creciente globalización a escala mundial de la 
economía, es decir, la continua ampliación de los mercados por la progresiva reducción de las barreras arancelarias, la desaparición paulatina de otros tipos de trabas al comercio internacional y la mejora de los transportes. Segundo, la difusión de la nueva tecnología electrónica, que a su enorme potencial de transformación de los métodos de producción y de gestión de las empresas une la posibilidad del procesamiento masivo de la información, con la informática, y su transmisión a distancia en tiempo real, con las telecomunicaciones.

En este marco se va configurando, frente al fordismo, un nuevo modelo de organización industrial, conocido como modelo de especialización flexible o, como prefieren otros autores, modelo de acumulación flexible (PIORE y SABEL, 1990; COSTA, 1990; MARTINELLI \& SCHOENBERGER, 1991; AMIN \& ROBINS, 1992). Éste consiste en la descentralización de la producción industrial mediante la segmentación de los procesos productivos en fases simples, cada una de las cuales, o varias de ellas agrupadas, se asignan a empresas o establecimientos distintos. Se ha ido pasando así de la factoría integral y la gran empresa fuertemente centralizada a la proliferación de talleres, fábricas y empresas marcadamente especializadas que forman redes empresariales complejas, mediante el establecimiento de relaciones de subcontratación, planteadas en términos de gran flexibilidad.

Este nuevo modelo se apoya en la tecnología de base electrónica: las nuevas máquinas son programables $y$, por tanto, polivalentes, es decir, capaces de desarrollar diversas tareas. Su uso permite fabricar a precios competitivos series pequeñas de productos y así atender satisfactoriamente demandas atomizadas, estacionales e incluso personalizadas. Las nuevas máquinas electrónicas son, por ello, más flexibles que las grandes cadenas electromecánicas y además requieren plazos de amortización mucho más cortos, por lo que con ellas resulta más fácil la adaptación continua de la empresa al cambio tecnológico.

Las nuevas tecnologías de telecomunicación, por su parte, hacen posible dirigir de manera coordinada la producción de varios establecimientos industriales alejados entre sí, lo cual permite elegir localizaciones distintas para cada uno de ellos, en función de las ventajas que cada emplazamiento tiene respecto a la fase del proceso productivo que se le asigna. Esta flexibilidad de localización queda reforzada por las continuas mejoras de los medios de transporte, que hacen cada vez más barata, más rápida y más segura la circulación de mercancías y, por tanto, resulta cada vez más fácil sincronizar la producción de varios establecimientos alejados entre sí.

La descentralización productiva y la consiguiente formación de redes de empresas explican dos hechos sobradamente constatados: primero, que las grandes empresas industriales han experimentado en las dos últimas décadas fuertes pérdidas de puestos de trabajo; segundo, que ha crecido notablemente el número de pequeñas y 
medianas empresas (PYME), gracias a las cuales ha sido posible absorber dichas pérdidas, total o parcialmente, según los casos. El nuevo modelo, por tanto, ha traído consigo una redistribución del empleo entre los tipos de empresa definidos según su tamaño.

Pero ésta no es sino una consecuencia de la aplicación al factor trabajo de los criterios de flexibilidad consustanciales al modelo. El concepto de flexibilidad laboral presenta dos acepciones distintas, que no se excluyen mutuamente, sino que se complementan, a las que cabe denominar, siguiendo a BENKO \& DUNFORD (1991), flexibilidad numérica y flexibilidad funcional.

La flexibilidad numérica hace referencia a la facilidad con que cuentan las empresas para ajustar el tamaño de sus plantillas a las fluctuaciones de la demanda y a las necesidades de la producción. La elevada concentración de obreros en las grandes factorías del modelo fordista trajo consigo el fortalecimiento de los sindicatos, que consiguieron para los trabajadores ciertas garantías respecto a las condiciones de trabajo y a la estabilidad en el mismo, garantías que fueron recogidas, de una $u$ otra forma, en la legislación laboral de los países industrializados.

Los empresarios, en las últimas décadas, han venido abogando por la reforma de dicha legislación, con el objetivo de alcanzar una flexibilidad numérica lo más alta posible, eliminando o reduciendo al máximo las garantías de estabilidad, que para ellos constituyen rigideces a la producción.

Las grandes empresas han ido reduciendo el tamaño de sus plantillas mediante la eliminación de trabajadores fijos por medio de despidos, bajas incentivadas o jubilaciones anticipadas. Si con posterioridad han necesitado incrementar la producción, han recurrido a la contratación temporal de trabajadòres, al "alquiler" de trabajadores a las empresas de trabajo temporal y, sobre todo, a la subcontratación de tareas a PYME especializadas. Así se explica la redistribución del empleo antes mencionada, por medio de la cual las grandes empresas han conseguido reducir sus costes laborales.

La flexibilidad funcional se refiere a la capacidad de los trabajadores para adaptarse a nuevas tareas productivas dentro de la empresa, en función de los cambios en la demanda, en la tecnología o en la política comercial. El fordismo consagró la figura del obrero como mero servidor de la máquina, cuyo trabajo se caracteriza por una marcada especialización en tareas concretas de gran simplicidad; como consecuencia de ello, el trabajador industrial no necesita más cualificación profesional que la que se adquiere con la práctica laboral, precedida, si acaso, de un corto adiestramiento. 
Ante la posibilidad de programar y reprogramar la maquinaria, con los consiguientes avances en la automatización de la producción industrial, el obrero servidor de la máquina es una figura cada vez menos útil en la empresa. Lo que ésta necesita ahora es un tipo de trabajador polivalente y flexible, dotado de unos conocimientos básicos amplios que le capaciten para comprender en su conjunto el proceso productivo y así poder desarrollar tareas diversas y adaptarse a los cambios e innovaciones. Para PIORE y SABEL (1990) se trata, en cierto modo, de un nuevo tipo de artesanado que se diferencia del tradicional en el grado de complejidad tecnológica.

Para la cualificación profesional de estos trabajadores no basta la mera práctica laboral, sino que se requiere una formación básica compleja, previa a la misma, y un frecuente reciclaje.

\section{CUALIFICACION PROFESIONAL Y LOCALIZACION INDUSTRIAL}

Como ya se ha adelantado, el modelo de especialización flexible tiene su reflejo en el espacio: el fordismo impulsó la concentración espacial de la industria en las ciudades, contribuyendo decisivamente al desarrollo de las grandes aglomeraciones urbano-industriales, mientras que el nuevo modelo permite una gran flexibilidad en la localización de los establecimientos, favoreciendo así la dispersión espacial de la industria.

Dada la tendencia a segmentar los procesos productivos y las posibilidades de coordinar el trabajo de varios establecimientos pertenecientes a la misma empresa o de varias empresas trabajando en régimen de subcontratación, aunque estén muy alejadas entre sí, las decisiones de localización pueden tomarse valorando ante todo las ventajas que cada territorio ofrece para cada fase concreta de la producción.

Entre dichas ventajas juega un papel muy importante el factor trabajo; de hecho, la búsqueda de la flexibilidad laboral por parte de las empresas ha dado lugar en las últimas décadas a un proceso de relocalización de indudable trascendencia. En los territorios donde hay salarios altos, una reglamentación laboral protectora de los trabajadores y una fuerte implantación sindical se han producido pérdidas importantes de empleos industriales, mientras que se han creado numerosos puestos de trabajo en territorios donde la mano de obra es barata y donde las empresas han encontrado menores trabas a la hora de aplicar la flexibilidad numérica.

Así se explican en gran parte los llamados procesos de industrialización periférica, es decir, el crecimiento rápido, en términos comparativos, del número de empresas y de empleos industriales en países del Tercer Mundo, en regiones débilmente 
industrializadas de los países desarrollados y en áreas del espacio rural (MÉNDEZ, 1987). Ésta es una de las vías por medio de las cuales han logrado las empresas reducir sus costes laborales y ganar competitividad.

Pero no todos los problemas de la producción industrial se resuelven con la flexibilidad numérica: para ciertos procesos productivos o ciertas fases de los mismos es imprescindible contar con trabajadores cualificados, los únicos capaces de garantizar la flexibilidad funcional que el nuevo modelo exige, siendo en este caso secundario el valor de los salarios. Los territorios que disponen de personal de estas características son los que tienen sistemas educativos bien desarrollados, que normalmente son los mismos que cuentan con una larga tradición industrial, pues cuanto mayor nivel de desarrollo se alcanza más se puede invertir en educación.

Teniendo esto en cuenta, la aparente desindustrialización de países y regiones desarrolladas o de grandes áreas metropolitanas cobra otra dimensión: lo que en realidad ha ocurrido es que de esos espacios se han marchado muchas empresas y establecimientos industriales dedicados a procesos productivos o fases de los mismos intensivos en mano de obra; pero se han quedado los que precisan personal cualificado, es decir, los de mayor complejidad tecnológica, los dedicados a tareas de investigación, innovación, planificación y gestión y, además, los servicios a la producción, a los que frecuentemente se hace referencia con el nombre de terciario industrial.

\section{LA SITUACION ESPAÑOLA: UNA BREVE INTRODUCCION}

Como respuesta a la transformación del modelo de organización de la industria, el gobierno español ha tomado en los últimos años decisiones importantes para incrementar la flexibilidad del factor trabajo: las novedades recientemente introducidas en la legislación laboral respecto a contratos temporales y a tiempo parcial o a empresas de trabajo temporal, así como la ampliación de las causas de despido, van orientadas a incrementar la flexibilidad numérica. Las reformas iniciadas en el terreno de la formación profesional a partir del Plan Nacional de Formación e Inserción Profesional de 1985, específicamente dedicado a la formación y reciclaje de la población activa, y a partir de la LOGSE, para la formación inicial de la población en edad escolar, tienen como finalidad incrementar la cualificación profesional de los trabajadores y, en consecuencia, mejorar la flexibilidad funcional.

Por lo que respecta a los agentes sociales, han tenido más trascendencia ante la opinión pública sus enfrentadas tomas de postura respecto a la flexibilidad numérica, rechazada por los sindicatos incluso con el recurso a la huelga general y defendida por 
los empresarios hasta el punto de considerar insuficiente la última reforma laboral, que su acuerdo básico en torno a la flexibilidad funcional, claramente puesto de manifiesto al menos en dos aspectos concretos: la reforma de las viejas ordenanzas laborales, que tiende a eliminar rigideces respecto a la asignación de funciones a los trabajadores dentro de las empresas, y el impulso a la formación continua de éstos.

Pero, aunque se siga trabajando en las dos direcciones, en el caso de España parece lógico poner el acento en la cualificación profesional: el elevado nivel de desarrollo económico del país, la larga tradición industrial de algunas regiones y el hecho de disponer desde hace tiempo de un sistema educativo que alcanza a la totalidad de la población infantil y juvenil son ventajas indudables de cara a la localización de empresas o establecimientos industriales de un cierto nivel tecnológico. Por el contrario, España no puede competir en cuanto a coste de la mano de obra con la mayor parte de los países de la Tierra, situación no lamentable, sino todo lo contrario, pues significa que se disfruta de un nivel de vida alto, al que no se debe renunciar de ningún modo.

Pero hoy en día presentan claras deficiencias estructurales tanto el sistema educativo, por lo que respecta a la formación profesional de los jóvenes, como el sistema de formación ocupacional, por lo que respecta a la población activa. Ello significa que España se enfrenta a graves dificultades a la hora de mejorar la cualificación del factor trabajo, lo cual entorpece el progreso industrial y el desarrollo económico del país.

Entre las publicaciones que realizan valoraciones de este tipo puede citarse un informe reciente del Consejo Superior de Cámaras de Comercio, Industria y Navegación de España: La formación profesional en el nuevo contexto europeo. En él se afirma que la corrección de los desequilibrios de la economía española se está viendo obstaculizada, entre otras cosas, por las deficiencias de la formación profesional: la escasez de personal cualificado en numerosos campos productivos "obliga a los gestores empresariales a una carrera por fichar a los empleados de sus competidores" (p. 17); la falta de reciclaje de los empleados menos cualificados "frena la movilidad funcional y geográfica, agravando aún más la segmentación del mercado de trabajo" (p. 17), a la vez que incrementa los gastos de protección por desempleo; la formación profesional que se imparte en los centros educativos está bastante disociada de la realidad laboral, por lo que, al acceder a un puesto de trabajo, los jóvenes han de recibir una formación complementaria en las empresas, lo cual puede calificarse como un despilfarro de recursos, privados y públicos.

Es fundamental, por tanto, superar estas deficiencias si no se quiere que la industria española siga perdiendo competitividad en los mercados internacionales, como se está poniendo de relieve en los últimos años. 


\section{UNA APORTACION EMPIRICA SOBRE LA RIOJA}

Entre los esfuerzos necesarios para superar esas deficiencias están los encaminados a adquirir un conocimiento preciso de las mismas y, en base a ello, realizar las propuestas oportunas. Con este fin, en los últimos años se han realizado en España estudios sobre la demanda de las empresas y la oferta de los trabajadores, en lo que respecta a cualificación profesional, así como sobre el grado de sintonía entre el sistema educativo y el mercado de trabajo.

El autor de este artículo ha dirigido un proyecto de investigación sobre esta temática, cuyo objetivo fue determinar los estudios de formación profesional que deben implantarse en cada una de las comarcas de La Rioja, teniendo en cuenta las necesidades laborales presentes y futuras de sus empresas, tanto las industriales como las de los demás sectores económicos ${ }^{1}$. La utilidad y oportunidad del proyecto se fundamentan en que, para desarrollar la LOGSE, se pondrán en marcha próximamente en todo el país los nuevos curricula de formación profesional y es importante acertar con los más adecuados a las necesidades de desarrollo de cada territorio. Si no se acierta, se habrá perdido una ocasión excepcionalmente favorable para superar las deficiencias actuales y remover uno de los obstáculos que entorpecen el desarrollo regional y comarcal.

La memoria final del proyecto incluye sendos análisis sobre la estructura económica de la región y cada una de sus comarcas, las necesidades de personal de las empresas y la oferta educativa de formación profesional existente. Para el estudio de las necesidades de personal se realizó una encuesta a una muestra de 204 empresas, seleccionada aleatoriamente y estratificada según tres criterios: el sector económico al que pertenecen, el tamaño de su plantilla y la comarca en que se localizan. El número de empresas encuestadas garantiza un margen de error inferior al $3 \%$, con un nivel de confianza del 95,5\%, mientras que la estratificación proporcional asegura la representatividad de la muestra. La encuesta se hizo en el segundo trimestre de 1993, por medio de entrevistas personales de los miembros del equipo de investigación, casi todos profesores de formación profesional, con los responsables de las empresas, sobre la base de un cuestionario.

En este artículo se va a hacer una explotación parcial de dicha encuesta: por un lado, centrándose exclusivamente en la industria, prescindiendo de los otros sectores económicos. Por otro lado, tomando en consideración sólo las preguntas del cuestionario que permiten detectar las actitudes de las empresas industriales respecto a la

1 El proyecto, ya concluido, se titula Estudio socio-laboral para un diseño de formación profesional específica en La Rioja y ha sido financiado por el Centro de Investigación, Documentación y Evaluación (CIDE) del Ministerio de Educación y Ciencia, a través de la convocatoria de ayudas a la investigación educativa del año 1992. 
cualificación profesional de sus empleados y las estrategias que adoptan de cara a la formación de los mismos, que es uno de los objetivos de este artículo.

\section{ACTITUDES Y ESTRATEGIAS DE LAS EMPRESAS INDUSTRIALES ANTE LA CUALIFICACION PROFESIONAL: EL CASO DE LA RIOJA}

En la tabla 1 se recogen los resultados de la encuesta más significativos para los propósitos de este artículo. Los niveles de cualificación profesional que en dicho cuadro se consideran son los establecidos por el Consejo de la Comunidad Económica Europea en 1985 (85/368/CEE), actualmente en vigor.

Los niveles son cinco, que corresponden a los grados de formación siguientes: el nivel 5 a una formación de ingeniero superior o licenciado universitario, el 4 a ingeniero técnico o diplomado universitario, el 3 a formación profesional superior o maestría, el 2 a formación profesional media u oficialía y el 1 a formación profesional básicamente práctica.

Lo primero que se observa en dicha tabla es que las plantillas de las empresas industriales de La Rioja se ajustan a una disposición aproximadamente piramidal: los trabajadores menos cualificados son el $61 \%$ del total, disminuyendo el porcentaje según se va subiendo de nivel, aunque los del 2 y 3 aparecen muy igualados (Fig. 1). Este es, por tanto, el punto de partida real: las empresas industriales riojanas tienen, en conjunto, unas plantillas escasamente cualificadas.

Las actitudes de las empresas ante esta situación quedan de manifiesto en sus respuestas sobre creación y reducción de puestos de trabajo para el futuro inmediato:

Por lo que respecta a la reducción, se repite la disposición piramidal antes señalada: el $54 \%$ de los puestos de trabajo a eliminar corresponden al nivel 1, el $27 \%$ al 2 y el $19 \%$ restante al 3; no va a desaparecer ninguno de los que equivalen a una formación universitaria (Fig. 1).

Respecto a la creación de puestos de trabajo, sin embargo, se invierte la situación: el $48 \%$ de los nuevos corresponderán al nivel 3, seguido del nivel 2 con un 20 $\%$ y el 4 con un $18 \%$. Al nivel 1 corresponderán sólo el 11 \% y al 5 el $3 \%$ (Fig. 1). 

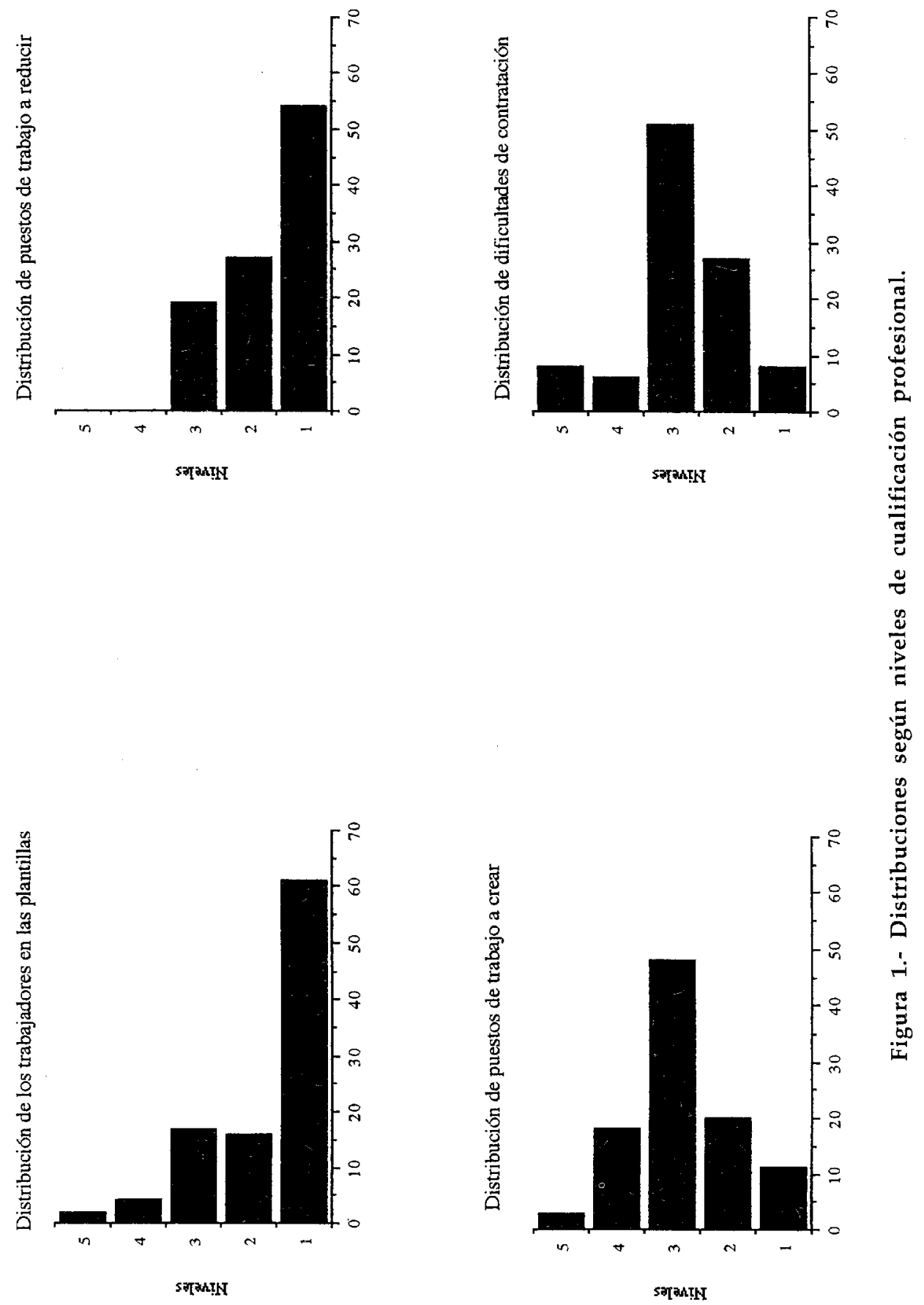
Tabla 1.- Distribución de puestos de trabajo por niveles de cualificación profesional en las empresas industriales de La Rioja (porcentajes).

\begin{tabular}{|l|r|r|r|r|c|}
\cline { 2 - 6 } \multicolumn{1}{c|}{} & \multicolumn{5}{c|}{ Niveles de cualificación profesional } \\
\hline Cuestiones planteadas * & $\mathbf{1}$ & $\mathbf{2}$ & $\mathbf{3}$ & $\mathbf{4}$ & $\mathbf{5}$ \\
\hline A: Trabajadores en plantilla & 61 & 16 & 17 & 4 & 2 \\
\hline B: Puestos de trabajo a reducir & 54 & 27 & 19 & 0 & 0 \\
\hline C: Puestos de trabajo a crear & 11 & 20 & 48 & 18 & 3 \\
\hline D: Dificultades de contratación & 8 & 27 & 51 & 6 & 8 \\
\hline
\end{tabular}

* El enunciado exacto de las preguntas formuladas en la encuesta es el siguiente:

A: ¿Qué nivel de cualificación poseen los operarios de su empresa?

B: ¿Qué puestos de trabajo van a reducirse a medio plazo en su empresa?

C: ¿Qué puestos de trabajo piensa crear a medio plazo en su empresa?

D: ¿Para cubrir qué puestos de trabajo ha encontrado recientemente dificultades de contratación?

Aunque la encuesta fue realizada en un momento de aguda recesión económica, proclive al pesimismo empresarial, el balance final entre las previsiones de creación y de eliminación de puestos de trabajo resulta positivo, es decir, los empresarios industriales riojanos preveían que sus plantillas se iban a incrementar. Pero es necesario matizar que ese balance resulta negativo para los trabajadores del nivel 1 , los de menor cualificación, y positivo para los demás, especialmente para los del nivel 3.

He aquí, pues, una primera conclusión significativa: los empresarios industriales de La Rioja tienen una actitud clara de mejorar la cualificación profesional de sus plantillas, actitud que se manifiesta en sus previsiones de creación y reducción de puestos de trabajo, pues aquélla se va a centrar casi exclusivamente en los niveles de cualificación intermedia, del 2 al 4, y ésta va a afectar mayoritariamente al 1, el de menor cualificación ${ }^{2}$. Esta actitud, a la luz de las reflexiones teóricas anteriores, sin duda resulta la correcta: sólo con trabajadores cada vez mejor cualificados se podrán abordar tareas de creciente complejidad tecnológica.

En otra de las preguntas de la encuesta se pidió a los empresarios que señalaran el nivel de cualificación de los trabajadores para cuya contratación en el año anterior

\footnotetext{
2 En un entorno empresarial dominado abrumadoramente por la PYME, como ocurre en La Rioja, es lógico que no exista apenas demanda de ingenieros superiores o licenciados universitarios.
} 
habían tenido dificultades, es decir, en que les había costado más tiempo del normal encontrar al candidato adecuado. El $51 \%$ de los casos corresponde al nivel 3 , el $27 \%$ al nivel 2 y el $8 \%$ al nivel 1 . Se trata, por tanto, de una disposición similar a la de la cuestión anterior (Fig. 1).

De aquí se extrae otra conclusión significativa: la actitud empresarial de mejora de la cualificación profesional de sus plantillas está tropezando con un obstáculo importante, que es la escasez de personal adecuadamente cualificado. Si este obstáculo no se remueve pronto, es decir, si no mejora sustancialmente la formación profesional de la población activa y de los jóvenes que se incorporan al mercado laboral, la correcta actitud empresarial de mejorar la cualificación de sus plantillas puede verse bloqueada.

La última pregunta de la encuesta que se va a comentar en este artículo es la siguiente: ¿Cuántos trabajadores han estado recibiendo formación profesional por cuenta de la empresa en los últimos años? Las respuestas dadas por los empresarios encuestados suman una cifra que no llega al $2 \%$ de las plantillas de sus empresas: 32 sobre un total de 1.794 .

La aparente contradicción entre la actitud manifiesta de mejorar la cualificación de sus plantillas y el escasísimo esfuerzo realizado en formación profesional no escapó a los miembros del equipo de investigación, que, mediante preguntas $u$ observaciones fuera de cuestionario, intentaron obtener de sus interlocutores algún elemento clarificador. La conclusión obtenida al respecto es que para incrementar la cualificación de sus plantillas laborales los empresarios optan mayoritariamente por contratar a personas cada vez mejor cualificadas, en vez de reciclar a sus propios trabajadores.

La adopción de esta estrategia se fundamenta en lo siguiente: primero, el temor a que los trabajadores reciclados por cuenta de la empresa la abandonen para colocarse en otra, aprovechando su perfeccionamiento personal, lo cual sería tanto como haber realizado una inversión para la competencia. Segundo, la convicción de que la formación no es tarea que corresponda a las empresas industriales, sino a otras instituciones: si aquéllas deben producir y lanzar al mercado productos adaptados a la demanda y competitivos en calidad y precio, éstas deben formar y colocar en el mercado laboral a trabajadores competentes, bien adaptados a las necesidades de las empresas industriales que demandan personal cualificado. 


\section{GENERALIZACION Y CONCLUSIONES}

Las conclusiones obtenidas en el estudio de La Rioja, por tanto, pueden resumirse así: las empresas industriales tienen unas plantillas con escasa proporción de personal cualificado, situación que los empresarios tienen previsto mejorar reduciendo los puestos de trabajo de menor cualificación y aumentando los de las tres cualificaciones intermedias; para ello acuden al mercado laboral, recurriendo escasamente al reciclaje de los trabajadores de plantilla, pero están teniendo problemas para encontrar el personal cualificado que necesitan.

Estudios realizados en otros ámbitos territoriales y con otras metodologías ${ }^{3}$, aunque no permitan comparaciones estadísticas rigurosas, sí que permiten afirmar que las actitudes y estrategias de los empresarios riojanos son similares a las de los empresarios de otras regiones, como Aragón, y del conjunto de España, por lo que estas conclusiones pueden generalizarse a dichos ámbitos territoriales.

A la luz de las reflexiones teóricas anteriores, cabe hacer una valoración negativa de esta situación, pues si bien la actitud de mejorar las cualificaciones profesionales por parte de los empresarios es, como se ha dicho antes, correcta, resulta difícil ponerla en práctica por falta de personal. Si esto no se corrige rápidamente puede degenerar en un auténtico bloqueo del desarrollo industrial futuro, lo cual sería muy grave para España, pues su industria se vería crecientemente desplazada de los mercados internacionales en los segmentos productivos que exigen mano de obra barata, como viene ocurriendo desde hace ya tiempo, y no podría tampoco competir en los segmentos de mayor complejidad tecnológica.

El resultado final podría ser el desmantelamiento paulatino de la industria nacional y la especialización económica del país en determinados servicios, de los que quedarían excluidos, obviamente, los que se agrupan en el terciario industrial antes aludido, que suelen ser servicios de muy alto valor añadido. Éste no es, indudablemente, el "escenario" deseable, aunque sí uno de los probables.

Para evitarlo hace falta un esfuerzo colectivo, tanto de la administración pública como de los trabajadores y empresarios. Hacen bien éstos en exigir a aquélla que se esfuerce en que al mercado laboral acudan trabajadores bien formados, pero no es una postura realista la de no invertir apenas en formación profesional: es significativo

\footnotetext{
3 Véanse, por ejemplo, el estudio Adecuación del sistema educativo a las necesidades del desarrollo regional, realizado por la Confederación Regional de Empresarios de Aragón, la Encuesta de requerimientos de empleo y formación en las empresas españolas, realizada por el Centro de Investigaciones Sociológicas, o el ya citado informe La formación profesional en el nuevo contexto europeo realizado por el Consejo Superior de Cámaras de Comercio, Industria y Navegación de España.
} 
a este respecto que mientras las empresas industriales españolas dedican a ello sólo el $0,2 \%$ de sus costes laborales, las alemanas dedican el 1,6 y las francesas el 1,8.

Por terminar en un tono optimista, cabe decir que el momento actual es esperanzador: la administración está trabajando en una ambiciosa reforma de la formación profesional, uno de cuyos elementos más positivos es la obligatoriedad de prácticas en las empresas. Los empresarios, cuyas organizaciones no sólo han acogido bien esta idea, sino que además se encuentran entre sus promotores ${ }^{4}$, acabarán cambiando su mentalidad actual y se implicarán en la reforma, que en el fondo les conviene, pues las mencionadas prácticas son un excelente medio para reclutar personal cualificado. Los sindicatos, por su parte, llevan años colaborando con la administración en la organización de cursos de perfeccionamiento para trabajadores, lo que, además de probar su actitud positiva al respecto, les ha permitido adquirir una valiosa experiencia.

\section{BIBLIOGRAFÍA}

Adecuación del sistema educativo a las necesidades del desarrollo regional. Confederación Regional de Empresarios de Aragón, 3 vol. Zaragoza, 1994.

AMIN, A. et ROBINS, K. (1992): Le retour des économies régionales? La géographie mythique de l'accumulation flexible. En: BENKO, G. et LIPIETZ, A. (Dir.): Les régions qui gagnent. Districts et réseaux: les nouveaux paradigmes de la géographie économique. 123-161. Presses Universitaires de France. Paris.

BENKO, G, \& DUNFORD, M. (1991): Structural change and the spatial organisation of the productive system: an introduction. En: BENKO, G, \& DUNFORD, M. (Ed.): Industrial change and regional development: the transformation of new industrial spaces. 3-23. Belhaven Press. London and New York.

COSTA, M.T. (1990): Los nuevos problemas de la organización industrial. En: VELARDE, J. GARCÍA DELGADO, J.L. y PEDREÑO, A. (Ed.): La industria española. Recuperación, estructura y mercado de trabajo. IV Jornadas de Alicante sobre Economía Española.155167, Colegio de Economistas de Madrid. Madrid.

Encuesta de requerimientos de empleo y formación en las empresas españolas. Coyuntura laboral. 34, 1-32, Centro de Publicaciones del Ministerio de Trabajo y Seguridad Social. Madrid, 1991.

\footnotetext{
${ }^{4}$ En 1984 las organizaciones empresariales firmaron los primeros acuerdos para la realización de prácticas en las empresas con centros privados de formación profesional.
} 
HALL, P. y PRESTON, P. (1990): La ola portadora. Nuevas tecnologías de la información y geografía de las innovaciones. 1846-2003. Fundación para el Desarrollo de la Función Social de las Comunicaciones, 323 p., Madrid.

La formación profesional en el nuevo contexto europeo. Consejo Superior de Cámaras de Comercio, Industria y Navegación de España, 180 p., Madrid, 1992.

MARTINELLI, F. \& SCHOENBERGER, E. (1991): Oligopoly is alive and well: notes for a broader discussion of flexible accumulation. En: BENKO, G, \& DUNFORD, M. (Ed.): Industrial change and regional development: the transformation of new industrial spaces. 117-133. Belhaven Press. London and New York.

MÉNDEZ, G. y DEL VALLE, R. (1987): Reestructuración productiva e industrialización periférica: claves para un debate. Boletín de la Asociación de Geógrafos Españoles. 5, 28-34, Asociación de Geógrafos Españoles. Tarragona.

PIORE, M.J. y SABEL, C.F. (1990): La segunda ruptura industrial. Alianza, 455 p., Madrid. 